\title{
Study on the Immobilization Technology of Hydroxyapatite in Heavy Metal Contaminated Sediment
}

\author{
Yunfei Li \\ Henan Medical College, Zhengzhou, Henan, China
}

\begin{abstract}
:
With the rapid development of industry, large amounts of untreated industrial waste water and domestic sewage carried heavy metal pollutants below into the water body with enrichment in sediments. When environmental conditions change, enrichment of heavy metals in sludge may be released into the overlying water causing the overlying water quality standard. The agent on immobilization of heavy metals in sludge is to be an extremely promising remediation technology in order to reduce impact on the environment. This test selects Hydroxyapatite and Nano Hydroxyapatite as curing agent and puts it into heavy metal pollution by different proportion. The paper conducts the research of curing agent and optimizes the better one. The paper selected HAP as matrix and $\mathrm{CaO}$ and $\mathrm{MgO}$ as different additives and studied complex condition of heavy metals in sediment of curing effect. Also the paper conduct the static releases test for pollutants in cured sediment in order to provide technical support for contaminated sediment remediation of heavy metal.
\end{abstract}

Keywords: Sediment; Heavy metals; Immobilization; Hydroxyapatite complex.

\section{INTRODUCTION}

On the complete water includes water, sediment and the surrounding environmental conditions. Sediments usually consist of little soil, sediment, organic matter and mineral mix, after a long time of biological, physical and chemical effects and the role of water transport and eventually deposited in formed by the bottom of the water, aquatic plant growth substrates and benthic animals thriving place, is an important part of aquatic ecosystems nutrient cycle. In recent years, with the development of industry, large amounts of untreated or non-conformance of sewage and industrial waste water carried pollutants into the water body. After pollutants into the water body, by adsorption of water particles, flocculation, sedimentation and biological absorption gradually deposited mud and accumulate. In the presence of continuous accumulation and enrichment, contaminants in the sediment concentrations tend to be much higher than the overlying water concentrations of pollutants. Of pollutants in sediment in the external environment conditions change may from sediment release again, cause the overlying water quality standard $[1,2]$. 
At this stage, as pollutants in sediments are mainly accumulated results, so the effects of sediment on overlying water quality is relatively durable, especially pollution basically put an end to the case, due to release pollutants in sediments overlying water caused by pollution have become water, especially the key factors of Lake water quality [3]. In water in the, end of mud and covered water exists with material and energy of exchange, end of mud in the pollution material and covered water in the pollution material exists with adsorption and solutions sucking of balance [4].

When water of environment status occurred change, end of mud in the of pollution material on has through this balance, from end of mud in the again release to Shang covered water in the, caused covered water deterioration, produced II times pollution, makes end of mud from pollution material of "meeting" into has pollution material of "source" [5].

Sediment contamination at this stage has become a major environmental problem in the world. Therefore, sediment pollution evaluation and treatment is an important content of water environment comprehensive improvement and can solve the problem of water pollution. Heavy metal is a specific gravity greater than 5 metals (generally refers to the density is greater than the $4.5 \mathrm{G} / \mathrm{cm} 3$ metals), but also refers to atomic weight metals greater than 55. By toxicity point, arsenic, selenium, boron and other elements are also included $[6,7]$.

Heavy metal pollution has the following features. (1) heavy metal and organic pollutants different, heavy metal cannot was biological activities decomposition, also impossible through burning of method clear, instead heavy metal will in organisms within rich set, part heavy metal also may through chemical reactions into became methyl compounds, caused more big against; (2) heavy metal pollution basic to composite sex pollution mainly, single elements pollution less, natural sources and human sources into end of mud are to organic, and inorganic of stick collection real of form into, This resulted in contaminated sediments are generally includes more than one combined pollution of heavy metals and (3) most have short distance migration of heavy metal or poor mobility features; (4) heavy metals on plants causing damage can lie dormant in the body for a long time, in the short term not easily found. (5) Heavy metals easily accumulate in the sediment [8].

Heavy metal and other of many pollutants different, it cannot was through microbial of life activities for decomposition, instead of, is can through series of role in biological body for rich set, so heavy metal as important of pollutants has larger of potential against sex heavy metal pollutants of source compared wide, it's in biological body residues time more long, easy in organisms within accumulation, and easily was found, serious effect to aquatic flora and fauna of growth and human of health [9]. Next, we describe the data and methodology used in more detail.

\section{MATERIAL AND METHODS}

Main source of heavy metals in sediment containing heavy metals in overlying water pollutants. Sources of heavy metals in water include the following: (1) geological and weathering. Baseline values and environmental background values are mainly from the role, but not to the exclusion of human impact. (2) Mining waste. Mainly comes from mines in the ore mining discharge into water bodies in the process of wastewater and waste rock piles and tailings after being washed into bodies of water, such as water pollution. (3) Industrial waste water. With the acceleration of industrialization, industrial wastewater mostly contains heavy metal contaminants produced in the production process, the issues in the oil refining, iron 
and steel, leather industry, fertilizer production, cable industry and electroplating industries, traditional industries particularly in production. (4) Domestic wastewater and urban runoff. Wastewater refers to unprocessed or just with a simple mechanical method of dealing with wastewater, which contains large amounts of pollutants. Adsorption of suspended particles in the urban atmosphere with a lot of heavy metal, under the action of gravity and sedimentation of suspended particles to the surface, with the formation of the precipitation and runoff into the water column. (5) Agricultural waste water. Chemical pesticides contain high levels of heavy metals in agricultural production, due to the massive use of chemical pesticides, resulting in farmland polluted by heavy metals, in the presence of rain into the water column.

$$
\begin{gathered}
b_{i j}^{\prime}=\frac{b_{i j}}{\sum_{i=1}^{n} b_{i j}} \\
\lambda_{\max }=\sum_{i=1}^{n} \frac{(B W)_{i}}{n W_{i}}
\end{gathered}
$$

Heavy metals in the sediments can in different ways combined with sediment composition, including: closed in amorphous materials; or adsorbed on the clay surface is hard oxidation state of iron in substance; in the lattices of carbonates, sulphates and oxides; and organic compound or in the lattice of silicate minerals. Because each has different forms of heavy metal is recovered, thus their bioavailability and toxicity have different impacts. So simply by the amount of heavy metals not provided accurate information about the characteristics of sediment pollution.

$$
\begin{gathered}
C I=\frac{\lambda-n}{n-1} \\
C R=\frac{C I}{R I}
\end{gathered}
$$

Due to the accumulation of heavy metals, when organism accumulates a certain number of victims of heavy metals will appear after the corresponding symptoms, affecting the development of organisms, or even cause the organism died. In place of heavy metal pollution, structure and function of aquatic ecosystems as a whole will suffer a devastating blow. Fish, shellfish and enrichment of heavy metals in water for human consumption, or heavy metals by aquatic plants (Lotus root) enrichment and human consumption, may enter the body of heavy metals, and enrichment in the human body. Heavy metal toxicity to humans mainly because of the heavy metals in the body and strong interactions in proteins, enzymes, resulting in loss of its activity or concentration in human organs and tissues for a long time, people have heavy metal poisoning, affect human health. In addition, some in normal physiology and metabolism of microorganisms, heavy metals can be transformed into more toxic substances, such as methyl Italy via the food chain bio magnification in the higher organisms progressively enriched levels of organisms in ecosystems of adverse reactions. Nonporous showed that copper in water, hard and heavy metals such as zinc can inhibit the growth of closterium lunular, and can influence the gender of fish in water and growth, and even lead to death.

$$
w=a_{1}, a_{2}, \cdots, a_{n}
$$

Similar to the contaminated soil remediation, sediment remediation technology of heavy metal pollution is divided into two basic. First is through dredging, the in-situ remediation technology in place to 
deal with the heavy metal, such as immobilization technology, phytoremediation,; the second is the dredging of contaminated bottom sediment light, regarding not ectopic remediation of sediments, such as leaching and flotation technologies.

$$
\begin{gathered}
\sum_{j=1}^{m} a_{j} b_{1 j}=b_{1} \\
\sum_{j=1}^{m} a_{j} b_{2 j}=b_{2} \\
\sum_{j=1}^{m} a_{j} b_{3 j}=b_{3}
\end{gathered}
$$

In situ fixation technique of heavy metal is mainly through the strengthening of heavy metals in bottom mud of adsorption, total precipitation and complexion abilities to improve the stability of heavy metals in sediments, thus reducing the migration of heavy metals, and toxicity and potential harm to the ecological environment. Because of the in-situ immobilization is, so the cost is relatively low. Worth noting is that immobilization of heavy metals in the sediments is only strengthened capacity, will not reduce the amount of heavy metals in sediments. Therefore, in some special cases (change of $\mathrm{pH}$, redox potential and changes), and some fixed heavy metal could be released into the water again, the overlying water secondary pollution.

Immobilization is the curing agent mixed with heavy metal contaminated sediments full, adjust and change the physical and chemical properties of heavy metals, through adsorption and complexion and precipitation, ion-exchange and oxidation-reduction reactions, the mobility of heavy metals in the environment, and significantly reduced the bioavailability, the heavy metal toxicity to plants and animals is reduced. Compared with the number of contaminated sediment remediation technologies, curing techniques can be used for in-situ remediation of heavy metals, can also be used for different repair, because of their high economic characteristics, more and more attention, and shown in the engineering application of a series of good results.

\subsection{Types and mechanism}

Immobilization of heavy metals remediation technology is the key to curing agent of choice. At this stage, common of curing agent main is divided into inorganic curing agent, and organic curing agent and complex distribution curing agent, which inorganic curing agent main has oxidation Han, and oxidation magnesium, and fly ash, alkaline material, ore, and acid hydrogen $\mathrm{f}$ beggar, and light base apatite, acid salts material and natural zeolite, and shoes algae soil, and expanded run soil, mineral; organic curing agent main has manure, and peat, and green manure, organic fertilizer,.

(1) Basic material types of curing agents. Oxidation of Korea, alkaline substances such as magnesium oxide as a curing agent for heavy metal pollution in sediment of its main mechanism for adjusting $\mathrm{pH}$ in sediments, changes in sediment of variable charge to enhance sediment adsorption for heavy metal ions, or is the formation of carbonate precipitation, effectively reducing the content of heavy metals in the bottom mud, reduce the toxicity of heavy metals contaminated sediments and bio-availability.

(2) Phosphate hardener. At this stage, light base gravy stones, o Han acid phosphate salt curing agent is considered effective, high performance curing agent. Acid salt-curing of heavy metals there are three main mechanisms: 1) phosphates of heavy metal adsorption on the surface; 2) heavy metal adsorption induced by acid salt; 3) phosphate react to form a precipitate or heavy metals and minerals. At present, the surrounding. Salt-curing soil contaminated by heavy metals and sediment nonporous certain progress has been made. 
(3) Natural mineral hardener. Zeolite, sepiolite and other natural mineral type curing mechanism of heavy metal is mainly because mineral particles as small, large specific surface area, full of negative charge on the surface and minerals and has a strong capability of adsorption and ion exchange.

(4) Organic stabilizer. Curing mechanism of heavy metal is mainly of farm manure and other organic matter hemic acids and hemic complex pollution of heavy metals ions in sediments, forming insoluble complexes, reducing toxicity and bio-availability of heavy metals.

(5) After curing. At present, heavy metal pollution in sediments, soils for a variety of combined pollution of heavy metals, taking into account the interaction between heavy metals, as well as for different metals of different curing effect of different tests and in practice is usually used after a variety of curing agent mixed to achieve efficient remediation of heavy metal contaminated results.

\subsection{Effect evaluation methods}

Total amount of heavy metals in sludge is evaluated for the determination of heavy metals in bottom mud of bioavailability and environmental effect of. Many studies have shown that, on the bioavailability of heavy metals in sediments and its environmental impact assessment, the total heavy metals alone cannot make good predictions. Studies show that potential impacts on the environment of heavy metals in sludge only the physic-chemical properties of the part, that part of the heavy metal is easily absorbed by biological, with potential implications for biological, so the need for heavy metal amount and sediment physicochemical properties of effective content for study. Heavy metal generally refers to the bioavailability of heavy metals in the environment are organisms absorb, accumulate or have toxic effects on the biological traits. Heavy metal bio-availability of information is an important means of contaminated sediment risk assessment, but also the management and remediation of contaminated sediments Foundation.

At present, the biological evaluation method for the effectiveness of many chemical extraction method is the most common major, followed by biological methods. Biological methods using ecological and toxicological analysis includes plant indication, microbiological evaluation and other chemical extraction method is the use of different chemicals to extract classified by morphology of heavy metals in the bottom mud, through more live in sediment physicochemical properties of that part of the contents of heavy metals, and thus to evaluate bioavailability. At this stage, chemical extraction procedure because of its efficient, economic characteristics, are widely used in the evaluation of heavy metal bio-availability. Chemical extraction method mainly has following several kinds. (1) A single extract. Single extraction reagent on extracting heavy metals in the sediments is called single extraction. Heavy metals to the environment caused by short-term or medium-term harm is usually evaluated by single extraction based on samples of different nature, composition, and extraction of heavy metals different kinds for different purposes, the extract of your choice will not be exactly the same. When extracting heavy metals of different extraction agent, according to their own nature of the integration agent can be divided into extraction of neutral salt, acid extraction method, and the infusion method. (2) The order of extraction. Sequential extraction method is based on a series of natural and artificial simulation of changes in environmental conditions, using different selective reagent, from weak to strong, continuous melting different absorption of trace elements in mineral phases. At present, widely used extraction methods with Tessier in the order of five-step extraction Tessier and EC standards Council based on BCR in the development of a three-step method.

Immobilization to repair part of the sediment contamination by heavy metals chemical remediation methods, which can be applied to in-situ remediation can also be applied to the different repair. There is no cost-effective means to remove the sediment directly, and repair technology of Chemical fixation method is 
an efficient and economical and can be applied to in-situ remediation, which will save you an expensive dredging costs. To reduce pollutants by means of chemical migration is an important way to reduce the risk of heavy metal pollution in the food chain. Immobilization of heavy metals is by adding organic matter, zeolite, phosphate exogenous substances, adjust and change the physical and chemical properties of heavy metal, to produce precipitation, adsorption, ion exchange, humiliation and oxidation-reduction reactions and reduce the bioavailability and mobility in the environment, thus reducing the heavy metal toxicity to plants and animals. People in need of a low cost and fast methods of in-situ remediation of heavy metal contaminated, in all of the in-situ remediation methods, repair technology of in-situ Chemical fixation method is better able to meet the requirements of controlling heavy metal pollution.

It is a measure of heavy metal bio-availability of heavy metals mobility and ecological impact of key parameters in ecological geochemical assessment plays an important role. Ministry of land and resources the ongoing new round of multipurpose regional geochemical survey and assessment, further attention on the bioavailability of heavy metals. Evaluation of effects of heavy metal pollution, mainly through the evaluation of repair of heavy metal bio-availability. Biological evaluation method for the effectiveness of more, depending on the object of study can be classified into three categories, namely direct or indirect physical, chemical and biological evaluation.

\section{RESULTS}

Sediments background value analysis indicators are $\mathrm{pH}$, water content, total phosphorus, heavy metals ( $\mathrm{Zn}, \mathrm{Mn}, \mathrm{Pb}$, and $\mathrm{Cd})$ and organic matter. Results are shown in table 1.

\section{TABLE I. Contaminated Sediment Physicochemical Properties}

\begin{tabular}{|l|c|}
\hline Parameter & Values \\
\hline water content & 29 \\
\hline $\mathrm{pH}$ & 8.2 \\
\hline total phosphorus & 1.5 \\
\hline $\mathrm{Zn}$ & 6865 \\
\hline $\mathrm{Mn}$ & 876 \\
\hline $\mathrm{Pb}$ & 298 \\
\hline $\mathrm{Cd}$ & 5.2 \\
\hline organic matter & 14.2 \\
\hline
\end{tabular}

(1) Sludge pre-treatment. Back to remove the upper layer of the sediment with water, rolled out in the tray, picking up shells, rocks, leaves and other debris. Sediment in ventilation, natural conditions losing cattle dried mud mortar grinding. (2) Sludge-determination of $\mathrm{pH}$ value. Take 5 grams of sediment samples placed in a small beaker, 1:5 Add deionized water using magnetic Range Rover with vigorous stirring 112 minutes, that sediment in the water completely dispersed, then rest for 30 minutes. Measurements of sediment samples with $\mathrm{pH}$ meter $\mathrm{pH}$ value, repeated three times and take the average. (3) Determination of organic matter in sediments. (4) Determination of total phosphorus in sediment. Digestion for determination of total phosphorus in sediment of a PIN spread anti-spectrophotometry. Weigh the amount of soil, add 
concentrated sulphuric and per chloric acid digestion, adding Noah $\mathrm{pH}$ regulating digestion fluid, to the solution is yellowish, then add Mo TI resistant reagent, 700nm wavelength using a spectrophotometer absorbance, are compared with the standard curve, phosphorus concentrations in the soil. (5) Determination of heavy metals in sediment. Determination of heavy metals in sediment using HN03-HC1-HF method.

Test by two big part composition, first part is curing agent of optimization research, main research common of curing agent on end of mud in the heavy metal of fixed of effect, optimization out effect best of curing agent, and determine optimal curing agent of best voted added volume; second part is complex distribution curing agent on heavy metal of fixed of research, main is through to filter out of curing agent in the joined additives get complex distribution curing agent, research complex distribution of curing agent on heavy metal of fixed of effect, and through curing end of mud in the pollutants of static release Institute GUI, Simulation investigation cured in sediment metal and total phosphorus in natural conditions to unleash that potential.

Sediment samples, respectively $1 \%, 5 \%$, and $10 \%$ to determine the effects of different application amount, respectively, sediment samples and mixes, 1:]0 add a second water, indoor place, stir once a day for 7 days, then continue to stand for 7 days, dried by quadrant sampling, put the samples in a sealed refrigeration, for follow-up testing. After curing mud extracted by BCR and the toxicity characteristic leaching procedure (TCLP) for curing effect, two parallel samples in each group, each test sediment quality as $50 \mathrm{~g}$, set the pure sediment samples for the control group, and the results averaged.

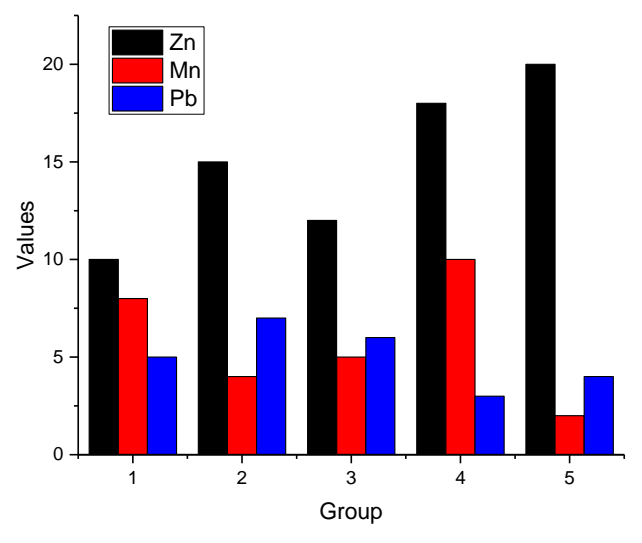

Fig. 1. Content Distribution of $\mathrm{Zn}$ in Sediments under Different Fertilizer Conditions for Curing Agent

Figure 1 shows that when the effect of curing agent of 1\%, on the whole, various curing agents for polluted sediments in the immobilization of $\mathrm{Zn}$ has some effect. Contrast can be found with the blank sample, imposing sample of curing agent, acid-extractable $\mathrm{Zn}$ content of 3\%-6\%, reducible $\mathrm{Zn}$ contents increase range is 3\%-6\%, oxidation States of $\mathrm{Zn}$ content and clear State of $\mathrm{Zn}$ residue content is small, only about $1 \%$ changes. Visible $1 \%$ of addition of curing agent to easily extract speciation work. When the effect of curing agent of 5\%, compared with the blank samples, acid-extractable $\mathrm{Zn}$ content has a certain amount of reduction. Application samples are compared with the control group of the nHAP, reduces the content of acid-extractable $\mathrm{Zn} 8 \%$, reducible and residual content of $\mathrm{Zn} \mathrm{5 \%}$ and $3 \%$ respectively, visible under the action of acid-extractable $\mathrm{Zn}$ in nHAP's transformation to reducible, a small amount of reducible $\mathrm{Zn}$ and oxidation States to clear residue transformation. Overall, the after effects of curing agent, which can make the acid-extractable $\mathrm{Zn}$ reducible to $\mathrm{Zn}$ into. When the hardener dosage as $10 \%$, Eva Dehydrogenase algae 
soil, can be seen Korean and carbonate sediments in the acid-extractable $\mathrm{Zn}$ was decreased to 9.92\%, 7.61\% and $7.38 \%$, accordingly, reduced $\mathrm{Zn}$ contents increase the $5.09 \%, 2.47 \%$ and $3.46 \%$, clear State of $\mathrm{Zn}$ residue content is to $34.08 \%, 35.62 \%$ and $34.02 \%$ respectively. But it can be seen that oxidized $\mathrm{Zn}$ content does not change much. HAP and nHAP acid-extractable $\mathrm{Zn}$ content reduced by $9.5 \%$ and $8.98 \%$, people clearly state $\mathrm{Zn}$ increases $5.97 \%$ and $5.03 \%$ can be oxidized $\mathrm{Zn}$ contents are not very clear.

Taking sediment samples cured using toxicity characteristic leaching procedure (TCLP) to evaluate the immobilization of heavy metals contaminated sediments. Figure 2 stabilizer under different fertilizer conditions the amount of heavy metals.

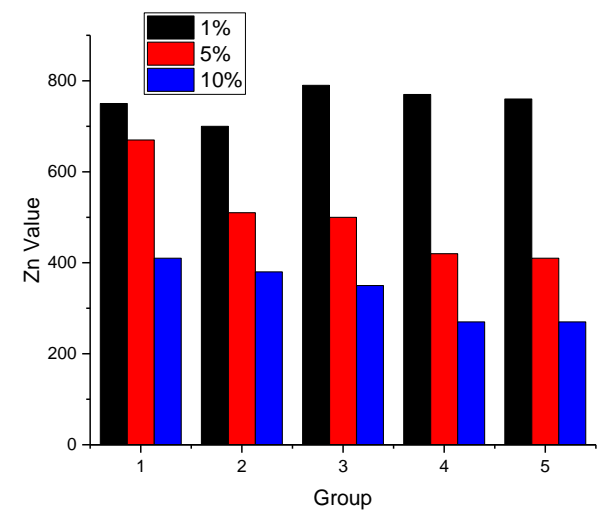

Fig. 2. Content Distribution of Mn in Sediments under Different Fertilizer Conditions for Curing Agent

As shown in Figure 2, not of imposing curing agent in sediment in the control group, the amount of $\mathrm{Mn}$ for 224 mg-kg. Imposed after the curing agent, the amount of Mn were reduced to a certain degree. When the hardener dosage as $1 \%$, slight decreases the amount of Mn. When the hardener dosage as 5\%, the amount of Mn began a significant decline. When the hardener dosage as 10\%, obvious from the diagram, $\mathrm{f}$ beggar carbonate, HAP and nHAP best for Mn immobilization in sediments, decreases in the amount of leaching of $\mathrm{Mn}$ and $25.01 \%$, and $23.66 \%$. No control group of imposing curing agent in sediment, the amount of $\mathrm{Pb}$ is 0.45 mg.kg. Imposed after the curing agent, has obvious effect on the amount of $\mathrm{Pb}$ in sediment, especially with hardener. As the amount of hardener to impose increased immobilization of $\mathrm{Pb}$ was more obvious. Dang curing agent added volume for $10 \%$ Shi, imposed baby algae soil and carbonate Bute of curing end of mud in the, $\mathrm{Pb}$ of dip out volume respectively for $1.03 \mathrm{mg}-\mathrm{kg}>0.89 \mathrm{mg}-\mathrm{kg}$, and blank group compared, respectively reduced has 50.72\% and 57.42\%; imposed phosphate II hydrogen Han, and $\mathrm{HAP}$ and nHAP, containing $\mathrm{p}$ curing agent of end of mud in the, $\mathrm{Pb}$ of dip out volume respectively for 0.42 mg.kg-i, and 0.45 mg-kg, 0.56mg-kg, and blank group compared, Reduced, and $79.9 \%$ and $73.21 \%$, respectively. Visible, phosphorus-containing curing agent for immobilization of $\mathrm{Pb}$ in sediment with good results. By comparing different in different fertilizer conditions on immobilization of heavy metals in sediments can be found, HAP and nHAP on curing effect of heavy metals in the sediments are more prominent, but nHAP prone to accumulation in the water, sediment mixed with hooks, leads to reduced effect. Meanwhile, higher cost of Nano-HAP, is three times the normal HAP, and between the two there is little difference between the curing effects of heavy metals in sediment, so use normal HAP as heavy metal pollution in sediments of curing agent. 


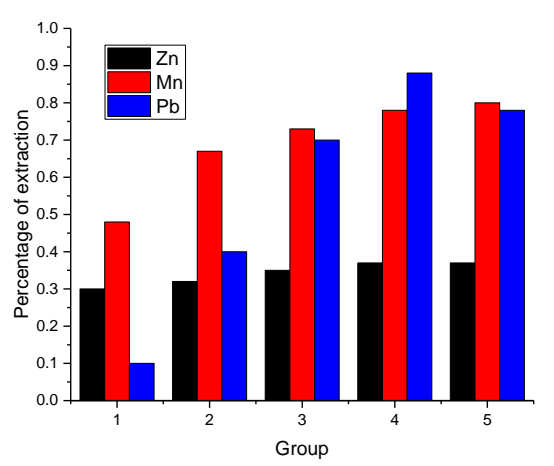

Fig. 3. Percentage of extraction of heavy metal in Sediments under Different Fertilizer Conditions for Curing Agent

Will by base apatite (HAP) by end of mud samples volume of 5\%, and $8 \%$, and $10 \%$, and $12 \%$, and $14 \%$ determine different application volume, respectively and end of mud samples full mixed, 1:10 added II times water, Yu indoor placed, daily mixing a times, continuous 7 days, then continues to static reset 7 days, dried used four points method sampling, will samples put Yu sealed bags in the refrigerated save, for followup test. After curing mud extracted by BCR method and effect evaluation of curing the TCLP procedure, each set of two parallel samples test sediment quality as the $50 \mathrm{~g}$ of each group, set the pure sediment samples for the control group, and the results averaged. Effect of different HAP on the morphology of heavy metals in the sediments of effects and fixed effects. As the HAP dosage increases, mud acid-extractable $\mathrm{Zn}$ content in a certain amount of reduction, oxidation state, and clearly state and other content has a certain increase of stable form. But when the hardener application rate is greater than $10 \%$, the reduction in acidextractable $\mathrm{Zn}$ and stable $\mathrm{Zn}$ increases less and less, and different forms of $\mathrm{Zn}$ contents in different fertilizer conditions not very obvious differences between each other. When HAP quantity is greater than $10 \%$, the amount of $\mathrm{Zn}$ in sediments in $220 \mathrm{mg}$, change is not obvious.

To sum up, the light base due to its structure, composition and other characteristics of apatite, the fixation mechanism of heavy metals including: heavy metal adsorption-induced, reacts with heavy metals deposition or minerals, directly on the surface to absorb heavy metals. Ensures maximum heavy metals in bottom mud of fixed and try to reduce the amount of hardener application, conditions to reduce project cost, select $10 \%$ as the HAP ratio during the curing process.

\section{CONCLUSIONS CONFLICT OF INTEREST ACKNOWLEDGMENT}

Studied of curing agent on immobilization of heavy metals pollution in sediment. Selection, and phosphate II hydrogen Han, and carbonate Han, and Tsunemoto apatite (HAP) and Nano by base apatite (nHAP) on heavy metal pollution end of mud in the of $\mathrm{Zn}$, and $\mathrm{Mn}$, and $\mathrm{Pb}$ and $\mathrm{Cd}$ four species heavy metal for fixed of, used BCR extraction method and TCLP method on curing effect for evaluation, hoof elected best curing agent, determine best application volume, and used SEM and FTIR on curing mechanism for analysis [10].

(1) by BCR sequential extraction method and the toxicity characteristic leaching procedure (TCLP) to 5 kinds of curing agents the immobilization of evaluation and analysis, can be found on the premise of equal amount, HAP and nHAP immobilization of heavy metals in sediment effect is superior to other curing 
agents. Taking into account the nHAP prone to accumulation in the water, mixed with sediment is not uniform, leads to reduced effect, while nHAP cost high, is three times the normal HAP and curing effect on heavy metals in the sediments of the two differ, so use normal HAP as heavy metal pollution in sediments of curing agent.

(2) On the premise of different dosage, HAP on immobilization of heavy metals in sediments was investigated effects. By comparing the test results, ensures maximum heavy metals in bottom mud of fixed and try to reduce the amount of hardener application, conditions to reduce project cost, select $10 \%$ as the HAP applied during the curing process. In this condition, $\mathrm{Zn}$, and $\mathrm{Mn}$, and $\mathrm{Pb}$ and Cd TCLP extraction volume reduced by $75.95 \%$, and $28.13 \%$, and $79.09 \%$, as well as steady-state (reducible, and clearly States) content, $7.61 \%, 7.97 \%$ and $4.17 \%$ respectively.

(3) Analysis by SEM and FTIR spectra and other scholars can be seen, HAP has a larger surface area, and contains a lot of CA, and the CA can be replaced by a series of metal ions, the fixation mechanism of heavy metals including: heavy metal adsorption-induced, reacts with heavy metals precipitation or minerals, directly on the surface to absorb heavy metals.

Curing agent on end of mud for in situ fixed, although part target heavy metal was fixed in end of mud in the, but has research showed that will improve some target outside of pollutants of release, and due to has new of material of introduced, whether will on original end of mud ecological system caused damage, whether on end of mud itself and Shang covered water caused other negative effect, also to be more in-depth of inquiry, need more full, and full of programme to validation. Immobilization of heavy metals in bottom mud of restoration is the restoration of a, its essence is to heavy metal from the unstable form into a stable form, are a constant number of heavy metals in the bottom mud. Curing effects of longevity and stability needed more in-depth inquiry [11].

We know of no previous register-based study that has illustrated the relevance of these two crucial issues in an equally detailed manner as we have done here.

\section{REFERENCES}

[1] Mulligan, C. N., R. N. Yong, and B. F. Gibbs. "Remediation technologies for metal-contaminated soils and groundwater: an evaluation." Engineering geology 60.1 (2001): 193-207.

[2] Ryan, James A., et al. "Method for in-situ immobilization of lead in contaminated soils, wastes, and sediments using solid calcium phosphate materials." U.S. Patent No. 5,512,702. 30 Apr. 1996.

[3] Wane, Raymond A., and Felix E. Okieimen. "Heavy metals in contaminated soils: a review of sources, chemistry, risks and best available strategies for remediation." ISRN Ecology 2011 (2011).

[4] Vangronsveld, Jaco, et al. "Phytoremediation of contaminated soils and groundwater: lessons from the field." Environmental Science and Pollution Research 16.7 (2009): 765-794.

[5] Basta, N. T., and S. L. McGowen. "Evaluation of chemical immobilization treatments for reducing heavy metal transport in a smelter-contaminated soil." Environmental pollution 127.1 (2004): 73-82.

[6] Bolan, Nanthi, et al. "Remediation of heavy metal (loid) s contaminated soils-to mobilize or to immobilize?." Journal of hazardous materials 266 (2014): 141-166.

[7] Seaman, John C., T. Meehan, and P. M. Bertsch. "Immobilization of cesium-137 and uranium in contaminated sediments using soil amendments." Journal of Environmental Quality 30.4 (2001): 1206-1213. 
[8] Guo, Guanlin, Qixing Zhou, and Lene Q. Ma. "Availability and assessment of fixing additives for the in situ remediation of heavy metal contaminated soils: a review." Environmental monitoring and assessment 116.13 (2006): 513-528.

[9] Wellman, Dawn M., Jonathan P. Icenhower, and Antoinette T. Owen. "Comparative analysis of soluble phosphate amendments for the remediation of heavy metal contaminants: Effect on sediment hydraulic conductivity." Environmental Chemistry 3.3 (2006): 219-224.

[10] Chiang, Yi Wai, et al. "Strategic selection of an optimal sorbent mixture for in-situ remediation of heavy metal contaminated sediments: framework and case study." Journal of environmental management 105 (2012): 1-11.

[11] Akcil, Ata, et al. "A review of approaches and techniques used in aquatic contaminated sediments: metal removal and stabilization by chemical and biotechnological processes." Journal of Cleaner Production 86 (2015): 24-36. 